\title{
Minimal Wave Speed in an Integrodifference System of Predator-Prey Type
}

\author{
Baoju Sun and Fuzhen Wu \\ Key Laboratory for Technology in Rural Water Management of Zhejiang Province, Department of Basic, \\ Zhejiang University of Water Resources and Electric Power Hangzhou, Zhejiang 310018, China
}

Correspondence should be addressed to Fuzhen Wu; fuzhwu@yeah.net

Received 6 April 2020; Accepted 20 May 2020; Published 8 June 2020

Academic Editor: Maria Alessandra Ragusa

Copyright (C) 2020 Baoju Sun and Fuzhen Wu. This is an open access article distributed under the Creative Commons Attribution License, which permits unrestricted use, distribution, and reproduction in any medium, provided the original work is properly cited.

\begin{abstract}
This article studies the minimal wave speed of traveling wave solutions in an integrodifference predator-prey system that does not have the comparison principle. By constructing generalized upper and lower solutions and utilizing the theory of asymptotic spreading, we show the minimal wave speed of traveling wave solutions modeling the invasion process of two species by presenting the existence and nonexistence of nonconstant traveling wave solutions with any wave speeds.
\end{abstract}

\section{Introduction}

Discrete time systems are widely used to model the evolution of species without overlapping generations, and some novel dynamics including chaos have been presented comparing with the corresponding continuous time models; see some models by Beddington et al. [1], Hofbauer et al. [2], and Weide et al. [3] and monographs by Murray [4]. One typical discrete time model takes the form as follows [2]:

$$
\begin{aligned}
u_{i, n+1} & =u_{i, n} e^{r_{i}-\sum_{j=1}^{K} a_{i j} u_{j, n},} \\
u_{i, 0} & >0,
\end{aligned}
$$

in which $K \in \mathbb{N}$ is a positive constant, $i \in\{1,2, \ldots, K\}$, $n \in \mathbb{N}$, and the other parameters are given. Although the form of (1) seems to be simple, it may be nonmonotone in its positive invariant region. For example, when $K=1$, we see [5]

$$
\begin{aligned}
u_{n+1} & =u_{n} e^{r\left(1-u_{n}\right)}, \\
u_{0} & >0
\end{aligned}
$$

has a positive invariant interval $[0, L]$ with

$$
L= \begin{cases}1, & r \in(0,1], \\ \frac{e^{r-1}}{r}, & r>1 .\end{cases}
$$

However, (2) is not monotone in the interval $[0, L]$ when $r>1$, which may reflect the phenomenon of overcompensatory growth in population dynamics (see Murray [4], Section 2.3). In the literature, the deficiency of monotonicity may lead to complex dynamics including the existence of nontrivial periodic solutions (the periodic solution is larger than 1) and other complex dynamics even in the scalar equations $[4,6,7]$. For coupled system (1), we may observe the plentiful dynamics by [1-3].

The energy transfer is one of the fundamental phenomena in the real world, and many predator-prey systems are established to model the process; we may refer to Murray [4] and Ruan [8] for some classical models and theoretical results. From (1), we may obtain different predator-prey systems by selecting proper parameters. In particular, after rescaling, one predator-prey system takes the form as follows: 


$$
\left\{\begin{array}{lll}
u_{n+1}=u_{n} e^{r_{1}\left(1-u_{n}-a v_{n}\right)}, & u_{0}>0, & n=0,1,2, \ldots, \\
v_{n+1}=v_{n} e^{r_{2}\left(1-v_{n}+b u_{n}\right)}, & v_{0}>0, & n=0,1,2, \ldots,
\end{array}\right.
$$

in which all the parameters are positive. Regarding (4) as a model in population dynamics, then $u_{n}, v_{n}$ stand for the densities of the prey and predator, respectively, $r_{1}>0, r_{2}>0$ depend on their intrinsic growth ratios, $a$ reflects the capture rate, while $b$ describes the energy conversion ratio. Similar to (2), we see that (4) may be nonmonotone since it does not admit the comparison principle appealing to the predatorprey system for some parameters. More precisely, we see that (4) has a positive invariant region $\left[0, L_{1}\right] \times\left[0, L_{2}\right]$ defined by

$$
\begin{aligned}
& L_{1}= \begin{cases}1, & r_{1} \in(0,1], \\
\frac{e^{r_{1}-1}}{r_{1}}, & r_{1}>1,\end{cases} \\
& L_{2}= \begin{cases}1+b L_{1}, & r_{2}\left(1+b L_{1}\right) \in(0,1], \\
\frac{e^{r_{2} b L_{1}+r_{2}-1}}{r_{2}}, & r_{2}\left(1+b L_{1}\right)>1 .\end{cases}
\end{aligned}
$$

Even if

$$
\left\{\begin{array}{l}
L_{1} \geq \bar{u}_{n+1} \geq \bar{u}_{n} e^{r_{1}\left(1-\bar{u}_{n}-a \underline{v}_{n}\right)} \\
L_{2} \geq \bar{v}_{n+1} \geq \bar{v}_{n} e^{r_{2}\left(1-\bar{v}_{n}+b \bar{u}_{n}\right)} \\
\underline{u}_{n+1} \leq \underline{u}_{n} e^{r_{1}\left(1-\underline{u}_{n}-a \bar{v}_{n}\right)} \leq L_{1}, \underline{v}_{n+1} \leq \underline{v}_{n} e^{r_{2}\left(1-\underline{v}_{n}+b \underline{u}_{n}\right)} \leq L_{2}, \\
\left(L_{1}, L_{2}\right) \geq\left(\bar{u}_{0}, \bar{v}_{0}\right) \geq\left(u_{0}, v_{0}\right) \geq\left(\underline{u}_{0}, \underline{v}_{0}\right) \geq(0,0),
\end{array}\right.
$$

for $n \in \mathbb{N}$, we cannot obtain

$$
\left(\bar{u}_{n}, \bar{v}_{n}\right) \geq\left(u_{n}, v_{n}\right) \geq\left(\underline{u}_{n}, \underline{v}_{n}\right), \quad n=1,2, \ldots,
$$

when

$$
\begin{array}{r}
r_{1} \in(0,1], \\
r_{2}\left(1+b L_{1}\right)<1,
\end{array}
$$

do not hold, which is similar to the possible nonmonotonicity in (2).

The evolutionary process of many species can be modeled by the integrodifference equations involving birthdiffusion process, in which birth and diffusion occur in different stages of individuals [9]. Some important examples of integrodifference equations can be found in Bourgeois et al. [10], Jacobsen et al. [11], Kot [12, 13], Lui [14], and a recent book by Lutcher [15]. Since Weinberger [16], the propagation dynamics of integrodifference equations has been widely studied, of which the abstract results can be applied to continuous time models [17]. In particular, Liang and Zhao [18] and Weinberger et al. [17] studied the propagation dynamics of monotone abstract integrodifference systems and applied the abstract results to several parabolic-type systems generating monotone semiflows. We also refer to Hsu and Zhao [19], Wang and Castillo-Chave [20], and Yi et al. [21] for some integrodifference systems that are local nonmonotone.

When the birth-diffusion process is concerned in (4), $\mathrm{Li}$ and $\mathrm{Li}$ [22] considered the following integrodifference system:

$$
\left\{\begin{array}{l}
u_{n+1}(x)=\int_{\mathbb{R}} u_{n}(y) e^{r_{1}\left(1-u_{n}(y)-a v_{n}(y)\right)} k_{1}(x-y) \mathrm{d} y, \quad x \in \mathbb{R}, n=0,1,2, \ldots, \\
v_{n+1}(x)=\int_{\mathbb{R}} v_{n}(y) e^{r_{2}\left(1-v_{n}(y)+b u_{n}(y)\right)} k_{2}(x-y) \mathrm{d} y, \quad x \in \mathbb{R}, n=0,1,2, \ldots,
\end{array}\right.
$$

in which $x \in \mathbb{R}, k_{1}, k_{2}$, which are called kernel functions, reflect the random walk of individuals, and monotone condition (8) holds. By direct calculation, when (8) is true, then (4) or (9) admits the comparison principle appealing to predator-prey systems and may have a coexistence steady state. When $k_{i}$ is of Gaussian type, $\mathrm{Li}$ and $\mathrm{Li}$ [22] established the existence of traveling wave solutions connecting $(0,0)$ with the coexistence state if the wave speed is larger than the threshold, which is finished by constructing upper and lower solutions.

Although $\mathrm{Li}$ and $\mathrm{Li}$ [22] investigated the traveling wave solutions of (9), there are some open problems for this system. Firstly, only the large wave speed is investigated, what is the threshold on the existence of nontrivial traveling wave solutions? Secondly, the movement law in [22] is too special, is it possible to consider the equation with more kernel functions? Thirdly, what will happen if (8) does not hold? The purpose of this paper is to further investigate the nontrivial (nonconstant) traveling wave solutions of (9) motivated by the above three questions. For example, we only make the following assumption on kernel functions:

(A) $k_{i}(x), x \in \mathbb{R}$, is Lebesgue-measurable and integrable such that $\int_{\mathbb{R}} k_{i}(x) \mathrm{d} x=1, k_{i}(x)=k_{i}(-x), x \in \mathbb{R}$, and there exists $\lambda>0$ such that $\int_{\mathbb{R}} k_{i}(y) e^{\lambda y} \mathrm{~d} y<$ $\infty, i=1,2$.

Evidently, (A) contains the Gaussian kernel as a special case. We also remove condition (8) to study the threshold that determines the existence or nonexistence of nontrivial traveling wave solutions for all positive wave speeds.

In Section 2, we shall give the definition and recall some known results. In Section 3, the existence of nonconstant traveling wave solutions is established by constructing generalized upper and lower solutions. Furthermore, we 
show the asymptotic behavior of traveling wave solutions and nonexistence of traveling wave solutions, which indicate a minimal wave speed of nontrivial traveling wave solutions. Finally, a further discussion is presented in Section 5.

\section{Preliminaries and Definitions}

In this paper, we use the standard partial ordering in $\mathbb{R}^{2}$. That is, if

$$
u=\left(u_{1}, u_{2}\right), v=\left(v_{1}, v_{2}\right) \in \mathbb{R}^{2},
$$

then

$$
u \leq v, \quad \text { iff } u_{1} \leq v_{1}, u_{2} \leq v_{2} .
$$

Moreover, $C(\mathbb{R}, \mathbb{R})$ denotes the set of uniformly continuous and bounded functions. When $b>a>0$, then

$$
C_{[a, b]}=\{u \in C: a \leq u(x) \leq b, x \in \mathbb{R}\},
$$

and $C^{+}$is defined by

$$
C^{+}=\{u \in C: u(x) \geq 0, x \in \mathbb{R}\} .
$$

We now recall the comparison principle and asymptotic spreading in Hsu and Zhao [19] and consider the initial value problem

$$
\begin{cases}w_{n+1}(x)=\int_{\mathbb{R}} b\left(w_{n}(y)\right) k(x-y) \mathrm{d} y, & x \in \mathbb{R}, n=0,1,2, \ldots \\ w_{0}(x)=w(x), & x \in \mathbb{R},\end{cases}
$$

in which $w(x) \in C^{+}, k$ satisfies $(\mathrm{A})$, and $b: \mathbb{R}^{+} \longrightarrow \mathbb{R}^{+}$such that

(B) There exists $B>0$ such that $b:[0, B] \longrightarrow[0, B]$ is continuous, and $\lim _{u \longrightarrow 0^{+}} b(u) / u:=b^{\prime}(0)>1$ satisfying

$$
0<b(u) \leq b^{\prime}(0) u, \quad u \in(0, B] .
$$

Lemma 1. If $w(x) \in C_{[0, B]}$, then (14) admits a unique solution $w_{n}(x) \in C_{[0, B]}, n \in \mathbb{N}$.

(1) Assume that $b:[0, B] \longrightarrow[0, B]$ is nondecreasing. If $\omega_{n}(x) \in C_{[0, B]}, n \in \mathbb{N}$, such that

$\left\{\begin{array}{l}\omega_{n+1}(x) \geq(\leq) \int_{\mathbb{R}} b\left(\omega_{n}(y)\right) k(x-y) \mathrm{d} y, \\ \omega_{0}(x) \geq(\leq) w(x),\end{array}\right.$

then $\omega_{n}(x) \geq(\leq) w_{n}(x), n \in \mathbb{N}, x \in \mathbb{R}$.

(2) Define

$$
c_{0}=\inf _{\lambda>0} \frac{\ln \left(b^{\prime}(0) \int_{\mathbb{R}} e^{\lambda y} k(y) \mathrm{d} y\right)}{\lambda} .
$$

(a) If $w(x) \in C_{[0, B]}$ admits nonempty compact support, then

$$
\underset{n \rightarrow \infty}{\limsup } \sup _{|x|>c n} u_{n}(x)=0, \quad c>c_{0} .
$$

(b) If $w(x) \in C_{[0, B]}$ admits nonempty support, then

$$
\limsup _{n \rightarrow \infty} \inf _{|x|>c n} u_{n}(x)>0, \quad c \in\left(0, c_{0}\right) .
$$

Remark 1. Due to the monotone condition, $\omega_{n}(x)$ is called an upper solution if we take $\geq$ in the first item of Lemma 1 , and we obtain a lower solution by taking $\leq$.

To study the traveling wave solutions, we first give the following definition. Here, a traveling wave solution of (9) is a special entire solution defined by

$$
\begin{aligned}
u_{n}(x) & =u(x+c n), \\
v_{n}(x) & =v(x+c n), \\
x & \in \mathbb{R}, \\
n & \in \mathbb{Z},
\end{aligned}
$$

in which $u, v \in C$ are the wave profile functions, while $c>0$ is the wave speed. Let $t=x+c n$; then, $u, v$, and $c$ must satisfy

$$
\begin{cases}u(t+c)=\int_{\mathbb{R}} u(y) e^{r_{1}(1-u(y)-a v(y))} k_{1}(t-y) \mathrm{d} y, & t \in \mathbb{R}, \\ v(t+c)=\int_{\mathbb{R}} v(y) e^{r_{2}(1-v(y)+b u(y))} k_{2}(t-y) \mathrm{d} y, & t \in \mathbb{R} .\end{cases}
$$

Similar to that in $\mathrm{Li}$ and $\mathrm{Li}$ [22], the purpose of this paper is to model that two species invade the same potential habitat, so $\phi, \psi$ satisfy

$$
\begin{aligned}
\lim _{t \longrightarrow-\infty} u(t) & =\lim _{t \longrightarrow-\infty} v(t)=0, \\
& \lim _{t \longrightarrow-\infty} \inf u(t)>\delta \\
t & \lim _{t \rightarrow-\infty} \inf v(t)>\delta
\end{aligned}
$$

for some $\delta>0$, of which the biological backgrounds are evident due to $c>0$ and $t=x+c n$. Thus, we need to investigate the existence of an integral system satisfying asymptotic boundary conditions.

In [23], Lin investigated the existence and asymptotic behavior of traveling wave solutions of integrodifference 
systems without monotone conditions. By Lin [23], Theorem 3.5, we present the following existence conclusion of (21).

Lemma 2. Assume that $\bar{u}(t), \underline{u}(t), \bar{v}(t), \underline{v}(t) \in C^{+}$such that

$$
\bar{u}(t), \underline{u}(t), \bar{v}(t), \underline{v}(t), \quad t \in \mathbb{R} .
$$

$$
\begin{aligned}
& \text { If } \\
& \bar{u}(t+c) \geq \int_{\mathbb{R}} u(y) e^{r_{1}(1-u(y)-a v(y))} k_{1}(t-y) \mathrm{d} y \geq \underline{u}(t+c), \\
& \bar{v}(t+c) \geq \int_{\mathbb{R}} v(y) e^{r_{2}(1-v(y)+b u(y))} k_{2}(t-y) \mathrm{d} y \geq \underline{v}(t+c),
\end{aligned}
$$

for all $t \in \mathbb{R}$ and any $u, v \in C^{+}$with

$$
\begin{gathered}
\bar{u}(t) \geq u(t) \geq \underline{u}(t), \\
\bar{v}(t) \geq v(t) \geq \underline{v}(t), \\
t \in \mathbb{R},
\end{gathered}
$$

then (21) has a solution $u^{*}(t), v^{*}(t) \in C$ such that

$$
\begin{gathered}
\bar{u}(t) \geq u^{*}(t) \geq \underline{u}(t), \\
\bar{v}(t) \geq v^{*}(t) \geq \underline{v}(t), \\
t \in \mathbb{R} .
\end{gathered}
$$

Remark 2. In Lemma 2, $(\bar{u}(t), \bar{v}(t))(\underline{u}(t), \underline{v}(t))$ are a pair of generalized upper and lower solutions of (21) without further monotone assumption. That is, the existence of (21) may be confirmed by the existence of generalized upper and lower solutions.

\section{Existence of Nonconstant Traveling Wave Solutions}

In this section, we shall show the existence of (21) by Lemma 2. Firstly, we define $c^{*}=\max \left\{c_{1}^{*}, c_{2}^{*}\right\}$ by

$$
c_{i}^{*}=\inf _{\lambda>0} \frac{r_{i}+\ln \left(\int_{\mathbb{R}} e^{\lambda y} k_{i}(y) \mathrm{d} y\right)}{\lambda} .
$$

Then, these constants satisfy the following lemma.

Lemma 3. $c_{1}^{*}>0, c_{2}^{*}>0$. Let

$$
\Lambda_{i}(\lambda, c)=e^{r_{i}} \int_{\mathbb{R}} e^{\lambda y-\lambda c} k_{i}(y) \mathrm{d} y, \quad i=1,2 .
$$

Then, they satisfy the following facts for $i=1,2$ :

(c1) If $c>c_{i}^{*}$, then $\Lambda_{i}(\lambda, c)=1$ has two positive roots $0<\lambda_{i 1}^{c}<\lambda_{i 2}^{c}<\infty$ such that $\Lambda_{i}(\lambda, c)<1$ with any $\lambda \in\left(\lambda_{i 1}^{c}, \lambda_{i 2}^{c}\right)$.

(c2) If $c \in\left(0, c_{i}^{*}\right)$, then $\Lambda_{i}(\lambda, c)>1, \lambda>0$.

(c3) If $c=c_{i}^{*}$, then $\Lambda_{i}(\lambda, c) \geq 1, \lambda>0$. Moreover, $\Lambda_{i}(\lambda, c)=1$ has a unique solution $\lambda_{i}^{c}$ such that

$$
\int_{\mathbb{R}}(y-c) e^{\lambda_{i}^{c}(y-c)} k_{i}(y) \mathrm{d} y=0 .
$$

By these positive constants, we show the following conclusion.

Theorem 1. For any fixed $c>c^{*},(21)$ has a positive solution $(u(t), v(t))$ such that

$$
\begin{aligned}
& \lim _{t \longrightarrow-\infty} u(t) e^{-\lambda_{11}^{c} t}, \\
& \lim _{t \longrightarrow-\infty} v(t) e^{-\lambda_{21}^{c} t},
\end{aligned}
$$

are positive.

Proof. We now show (21) has a pair of generalized upper and lower solutions. Let $\epsilon>0$ and $\eta>1$ such that

$$
\begin{aligned}
\epsilon & <\min \left\{\lambda_{11}^{c}, \lambda_{21}^{c}\right\}, \\
\Lambda_{i}\left(\lambda_{i 1}^{c}+\epsilon, c\right) & <1, \quad i=1,2, \\
\eta \lambda_{i 1}^{c} & <\min \left\{\lambda_{i 2}^{c}, \lambda_{i 1}^{c}+\epsilon\right\}, \quad i=1,2, \\
\Lambda_{2}\left(\eta \lambda_{21}^{c}+\epsilon, c\right) & <\infty .
\end{aligned}
$$

Define continuous functions

$$
\begin{aligned}
& \bar{u}(t)=\min \left\{L_{1} e^{\lambda_{11}^{c} t}, L_{1}\right\}, \\
& \bar{v}(t)=\min \left\{L_{2} e^{\lambda_{21}^{c} t}+q e^{\eta \lambda_{21}^{c} t}, L_{2}\right\}, \\
& \underline{u}(t)=\max \left\{L_{1} e^{\lambda_{11}^{c} t}-p e^{\eta \lambda_{11}^{c} t}, 0\right\}, \\
& \underline{v}(t)=\max \left\{L_{2} e^{\lambda_{21}^{c} t}-p e^{\eta \lambda_{21}^{c} t}, 0\right\},
\end{aligned}
$$

for $t \in \mathbb{R}$, where $p>L_{1}+L_{2}$ and $q>1$ are positive constants clarified later. Evidently, if $p$ and $q$ are large, then we have

$$
(\underline{u}(t), \underline{v}(t)) \leq(\bar{u}(t), \bar{v}(t)), \quad t \in \mathbb{R} .
$$

Moreover, it is clear that

$$
\begin{aligned}
\bar{u}(t) & \leq L_{1} e^{\epsilon t}, \\
\bar{v}(t) & \leq L_{2} e^{\epsilon t}+q e^{\epsilon t}, \\
t & \in \mathbb{R} .
\end{aligned}
$$

For any fixed $u(t), v(t) \in C$ with

$$
(\underline{u}(t), \underline{v}(t)) \leq(u(t), v(t)) \leq(\bar{u}(t), \bar{v}(t)), \quad t \in \mathbb{R},
$$

we first verify that

$$
\bar{u}(t+c) \geq \int_{\mathbb{R}} u(y) e^{r_{1}(1-u(y)-a v(y))} k_{1}(t-y) \mathrm{d} y, \quad t \in \mathbb{R},
$$

which is clear if $\bar{u}(t+c)=L_{1}$. Otherwise, we have 


$$
\begin{aligned}
\int_{\mathbb{R}} u(y) e^{r_{1}(1-u(y)-a v(y))} k_{1}(t-y) \mathrm{d} y & \leq e^{r_{1}} \int_{\mathbb{R}} u(y) k_{1}(t-y) \mathrm{d} y \\
& \leq L_{1} e^{r_{1}} \int_{\mathbb{R}} e^{\lambda_{11}^{c} y} k_{1}(t-y) \mathrm{d} y \\
& =L_{1} e^{\lambda_{11}^{c}(t+c)}=\bar{u}(t+c) .
\end{aligned}
$$

$$
\begin{aligned}
& \text { If } \bar{v}(t+c)=L_{2} \text {, then it is clear that } \\
& \qquad \bar{v}(t+c) \geq \int_{\mathbb{R}} v(y) e^{r_{2}(1-v(y)+b u(y))} k_{2}(t-y) \mathrm{d} y .
\end{aligned}
$$

Otherwise, $t<-c$, and we have $u(y) \leq L_{1} e^{\epsilon y}, y \in \mathbb{R}$, and there exists some constant $L>0$ such that

$$
\begin{aligned}
e^{b u}-1 \leq & L u, \quad u \in\left[0, L_{1}\right], \\
\int_{\mathbb{R}} v(y) e^{r_{2}(1-v(y)+b u(y))} k_{2}(t-y) \mathrm{d} y \leq & \int_{\mathbb{R}} v(y) e^{r_{2}(1+b u(y))} k_{2}(t-y) \mathrm{d} y \\
\leq & e^{r_{2}} \int_{\mathbb{R}} v(y) k_{2}(t-y) \mathrm{d} y+L e^{r_{2}} \int_{\mathbb{R}} u(y) v(y) k_{2}(t-y) \mathrm{d} y \\
\leq & e^{r_{2}} \int_{\mathbb{R}} \bar{v}(y) k_{2}(t-y) \mathrm{d} y+L e^{r_{2}} \int_{\mathbb{R}} \bar{u}(y) \bar{v}(y) k_{2}(t-y) \mathrm{d} y \\
\leq & e^{r_{2}} \int_{\mathbb{R}}\left[L_{2} e^{\lambda_{21}^{c} y}+q e^{\eta \lambda_{21}^{c} y}\right] k_{2}(t-y) \mathrm{d} y \\
& +L L_{1} e^{r_{2}} \int_{\mathbb{R}} e^{\varepsilon y}\left[L_{2} e^{\lambda_{21}^{c} y}+q e^{\eta \lambda_{21}^{c} y}\right] k_{2}(t-y) \mathrm{d} y \\
= & L_{2} e^{\lambda_{21}^{c}(t+c)}+q e^{\eta \lambda_{21}^{c}(t+c)} \Lambda_{2}\left(\eta \lambda_{21}^{c}, c\right) \\
& +L L_{1} e^{\left(\lambda_{21}^{c}+\varepsilon\right)(t+c)} \Lambda_{2}\left(\varepsilon+\lambda_{21}^{c}, c\right)+L L_{1} q e^{\left(\eta \lambda_{21}^{c}+\varepsilon\right)(t+c)} \Lambda_{2}\left(\varepsilon+\eta \lambda_{21}^{c}, c\right) .
\end{aligned}
$$

Thus, it is sufficient to verify that there exists $q>1$ large enough (such that $-t>0$ is large enough) such that

$$
\begin{aligned}
& L L_{1} e^{\left(\lambda_{21}^{c}+\epsilon\right)(t+c)} \Lambda_{2}\left(\epsilon+\lambda_{21}^{c}, c\right)+L L_{1} q e^{\left(\eta \lambda_{21}^{c}+\epsilon\right)(t+c)} \Lambda_{2}\left(\epsilon+\eta \lambda_{21}^{c}, c\right) \\
& \leq q e^{\eta \lambda_{21}^{c}(t+c)}\left(1-\Lambda_{2}\left(\eta \lambda_{21}^{c}, c\right)\right)
\end{aligned}
$$

which is true provided that

$$
\begin{array}{r}
2 L L_{1} e^{\left(\lambda_{21}^{c}+\epsilon\right)(t+c)} \Lambda_{2}\left(\epsilon+\lambda_{21}^{c}, c\right) \leq q e^{\eta \lambda_{21}^{c}(t+c)}\left(1-\Lambda_{2}\left(\eta \lambda_{21}^{c}, c\right)\right), \\
2 L L_{1} q e^{\left(\eta \lambda_{21}^{c}+\epsilon\right)(t+c)} \Lambda_{2}\left(\epsilon+\eta \lambda_{21}^{c}, c\right) \leq q e^{\eta \lambda_{21}^{c}(t+c)}\left(1-\Lambda_{2}\left(\eta \lambda_{21}^{c}, c\right)\right),
\end{array}
$$

when $q e^{\eta \lambda_{21}^{c}(t+c)}<L_{2}$. These inequalities are evident for $q>1$ large enough. After this, we may fix $q>1$ that is independent on $p$.

When $\underline{u}(t+c)=0$, it is clear that

$$
\int_{\mathbb{R}} u(y) e^{r_{1}(1-u(y)-a v(y))} k_{1}(t-y) \mathrm{d} y \geq \underline{u}(t+c) .
$$

If $\underline{u}(t+c)=L_{1} e^{\lambda_{11}^{c}(t+c)}-p e^{\eta \lambda_{11}^{c}(t+c)}>0$, then $t+c<0$ by $q>1$, and there exists $K>0$ such that

$$
\begin{aligned}
& \left|e^{r_{1}(1-u-a v)}-e^{r_{1}}\right| \leq K(u+v) \\
& u \in\left[0, L_{1}\right] \text {, } \\
& v \in\left[0, L_{2}\right] \text {, } \\
& \int_{\mathbb{R}} u(y) e^{r_{2}(1-v(y)+b u(y))} k_{2}(t-y) \mathrm{d} y \geq \int_{\mathbb{R}} v(y) e^{r_{2}(1-v(y))} k_{2}(t-y) \mathrm{d} y \\
& \geq \int_{\mathbb{R}} \underline{v}(y) e^{r_{2}(1-\bar{v}(y))} k_{2}(t-y) \mathrm{d} y \\
& \geq e^{r_{2}} \int_{\mathbb{R}} \underline{v}(y) k_{2}(t-y) \mathrm{d} y-A e^{r_{2}} \int_{\mathbb{R}} \underline{v}(y) \bar{v}(y) k_{2}(t-y) \mathrm{d} y \\
& \geq e^{r_{2}} \int_{\mathbb{R}} \underline{v}(y) k_{2}(t-y) \mathrm{d} y-A L_{2}(1+q) e^{r_{2}} \int_{\mathbb{R}} \underline{v}(y) e^{\varepsilon y} k_{2}(t-y) \mathrm{d} y \\
& =L_{2} e^{\lambda_{21}^{c}(t+c)}-p \Lambda_{2}\left(\eta \lambda_{21}^{c}, c\right) e^{\eta \lambda_{21}^{c}(t+c)}-A L_{2}(1+q) \Lambda_{2}\left(\eta \lambda_{21}^{c}+\varepsilon, c\right) e^{\left(\lambda_{21}^{c}+\varepsilon\right)(t+c)} \\
& \geq L_{2} e^{\lambda_{21}^{c}(t+c)}-p e^{\eta \lambda_{21}^{c}(t+c)}
\end{aligned}
$$


if

$$
\begin{aligned}
& p\left(1-\Lambda_{1}\left(\eta \lambda_{11}^{c}, c\right)\right) e^{\eta \lambda_{11}^{c}(t+c)} \\
& \quad \geq K\left(L_{1}+L_{2}+q\right) \Lambda_{1}\left(\eta \lambda_{11}^{c}+\epsilon, c\right) e^{\eta \lambda_{11}^{c}(t+c)}
\end{aligned}
$$

which is clear if $p>L_{1}$ is large.
When $\underline{v}(t+c)=0$, it is clear that

$$
\int_{\mathbb{R}} v(y) e^{r_{2}(1-v(y)+b u(y))} k_{2}(t-y) \mathrm{d} y \geq \underline{v}(t+c) .
$$

When $\underline{v}(t+c)=L_{2} e^{\lambda_{21}^{c}(t+c)}-p e^{\eta \lambda_{21}^{c}(t+c)}>0$, then $t+c<$ 0 , and there exists $A>0$ such that

$$
\begin{aligned}
0 & \leq e^{r_{2}}-e^{r_{2}(1-v)} \leq A v, \quad v \in\left[0, L_{2}\right] \\
\int_{\mathbb{R}} v(y) e^{r_{2}(1-v(y)+b u(y))} k_{2}(t-y) \mathrm{d} y & \geq \int_{\mathbb{R}} v(y) e^{r_{2}(1-v(y))} k_{2}(t-y) \mathrm{d} y \\
& \geq \int_{\mathbb{R}} \underline{v}(y) e^{r_{2}(1-\bar{v}(y))} k_{2}(t-y) \mathrm{d} y \\
& \geq e^{r_{2}} \int_{\mathbb{R}} \underline{v}(y) k_{2}(t-y) \mathrm{d} y-A e^{r_{2}} \int_{\mathbb{R}} \underline{v}(y) \bar{v}(y) k_{2}(t-y) \mathrm{d} y \\
& \geq e^{r_{2}} \int_{\mathbb{R}} \underline{v}(y) k_{2}(t-y) \mathrm{d} y-A L_{2}(1+q) e^{r_{2}} \int_{\mathbb{R}} \underline{v}(y) e^{\epsilon y} k_{2}(t-y) \mathrm{d} y \\
& \geq e^{r_{2}} \int_{\mathbb{R}}\left[L_{2} e^{\lambda_{21}^{c} y}-p e^{\eta \lambda_{21}^{c} y}\right] k_{2}(t-y) \mathrm{d} y-A L_{2}(1+q) e^{r_{2}} \int_{\mathbb{R}} e^{\left(\lambda_{21}^{c}+\epsilon\right) y} k_{2}(t-y) \mathrm{d} y \\
& =L_{2} e^{\lambda_{21}^{c}(t+c)}-p \Lambda_{2}\left(\eta \lambda_{21}^{c}, c\right) e^{\eta \lambda_{21}^{c}(t+c)}-A L_{2}(1+q) \Lambda_{2}\left(\eta \lambda_{21}^{c}+\epsilon, c\right) e^{\left(\lambda_{21}^{c}+\epsilon\right)(t+c)} \\
& \geq L_{2} e^{\lambda_{21}^{c}(t+c)}-p e^{\eta \lambda_{21}^{c}(t+c)} .
\end{aligned}
$$

if $p>1$ is large. The proof is complete.

Theorem 2. Assume that $k_{1}(y), k_{2}(y)$ have nonempty compact supports, respectively.

(1) If $c=c_{1}^{*}>c_{2}^{*}$, then (21) has a positive solution $(u(t), v(t))$ such that

$$
\frac{\lim _{t \longrightarrow-\infty} u(t) e^{-\lambda_{1}^{c} t}}{(-t)}, \lim _{t \longrightarrow-\infty} v(t) e^{-\lambda_{21}^{c} t} .
$$

are positive.

(2) If $c=c_{2}^{*}>c_{1}^{*}$, then (21) has a positive solution $(u(t), v(t))$ such that

$$
\begin{gathered}
\lim _{t \longrightarrow-\infty} u(t) e^{-\lambda_{11}^{c} t}, \\
\frac{\lim _{t \longrightarrow-\infty} v(y) e^{-\lambda_{2}^{c} t}}{-t},
\end{gathered}
$$

are positive.

(3) If $c=c_{1}^{*}=c_{2}^{*}$, then (21) has a positive solution $(u(t), v(t))$ such that

$$
\begin{gathered}
\frac{\lim _{t \longrightarrow-\infty} u(t) e^{-\lambda_{1}^{c} t}}{-t}, \\
\frac{\lim _{t \longrightarrow-\infty} v(t) e^{-\lambda_{2}^{c} t}}{-t},
\end{gathered}
$$

are positive.

Proof. We now prove the result by constructing generalized upper and lower solutions, which is similar to that in $[24,25]$. By the assumption, there exists $S>0$ such that

$$
\begin{aligned}
k_{i}(y) & =0, \\
|y| & >S+2 c .
\end{aligned}
$$

We first prove (1). Let $L>0$ such that $(-t+L) e^{\lambda_{1}^{c} t}, t<0$, is strictly increasing and

$$
\begin{aligned}
\left(-t_{1}+L\right) e^{\lambda_{1}^{c_{1}} t_{1}} & =L_{1}, \\
t_{1} & <-(S+2 c) .
\end{aligned}
$$

Define continuous functions

$$
\begin{aligned}
& \bar{u}(t)= \begin{cases}(-t+L) e^{\lambda_{1}^{c} t}, & t<t_{1}, \\
L_{1}, & t \geq t_{1},\end{cases} \\
& \underline{u}(t)= \begin{cases}(-t-p \sqrt{-t}) e^{\lambda_{1}^{c} t}, & t<-p^{2}, \\
0, & t \geq-p^{2},\end{cases} \\
& \bar{v}(t)=\min \left\{L_{2} e^{\lambda_{21}^{c} t}+q e^{\eta \lambda_{21}^{c} t}, L_{2}\right\}, \\
& \underline{v}(t)=\max \left\{L_{2} e^{\lambda_{21}^{c} t}-p e^{\eta \lambda_{21}^{c} t}, 0\right\},
\end{aligned}
$$

for some $p>0, q>L_{2}, \eta>1$. Evidently, we have

$$
(\underline{u}(t), \underline{v}(t)) \leq(\bar{u}(t), \bar{v}(t)), \quad t \in \mathbb{R},
$$

if $p$ and $q$ are large. We now confirm that they are generalized upper and lower solutions of (21) if $p$ and $q$ are large, and the desirable inequalities are clear if they are constants. 
For any fixed $u(t), v(t) \in C$ with

$$
(\underline{u}(t), \underline{v}(t)) \leq(u(t), v(t)) \leq(\bar{u}(t), \bar{v}(t)), \quad t \in \mathbb{R},
$$

we first verify that

$$
\bar{u}(t+c) \geq \int_{\mathbb{R}} u(y) e^{r_{1}(1-u(y)-a v(y))} k_{1}(t-y) \mathrm{d} y, \quad t \in \mathbb{R} .
$$

By the definition of $L>0$, we see that $\bar{u}(t+c)<L_{1}$ implies $-t_{1}>S+2 c$, and

$$
\begin{aligned}
& \int_{\mathbb{R}} u(y) e^{r_{1}(1-u(y)-a v(y))} k_{1}(t-y) \mathrm{d} y \\
& \leq e^{r_{1}} \int_{\mathbb{R}} u(y) k_{1}(t-y) \mathrm{d} y \\
& \leq L_{1} e^{r_{1}} \int_{\mathbb{R}}(-y+L) e^{\lambda_{1}^{c} y} k_{1}(t-y) \mathrm{d} y \\
& =L_{1}(-(t+c)+L) e^{\lambda_{1}^{c}(t+c)}=\bar{u}(t+c) .
\end{aligned}
$$

In the similar way, we may verify other inequalities. In case (2), we define continuous functions

$$
\begin{aligned}
& \bar{v}(t)= \begin{cases}(-t+L \sqrt{-t}) e^{\lambda_{2}^{c} t}, & t<t_{2}, \\
L_{2}, & t \geq t_{2},\end{cases} \\
& \underline{v}(t)= \begin{cases}(-t-p \sqrt{-t}) e^{\lambda_{2}^{c} t}, & t<-p^{2}, \\
0, & t \geq-p^{2},\end{cases} \\
& \bar{u}(t)=\min \left\{L_{1} e^{\lambda_{11}^{c} t}, L_{1}\right\}, \\
& \underline{u}(t)=\max \left\{L_{1} e^{\lambda_{11}^{c} t}-p e^{\eta \lambda_{11} t}, 0\right\},
\end{aligned}
$$

by similar parameters in case (1). For case (3), we define

$$
\begin{aligned}
& \bar{u}(t)= \begin{cases}(-t+L) e^{\lambda_{1}^{c} t}, & t<t_{1}, \\
L_{1}, & t \geq t_{1},\end{cases} \\
& \underline{u}(t)= \begin{cases}(-t-p \sqrt{-t}) e^{\lambda_{1}^{c} t}, & t<-p^{2}, \\
0, & t \geq-p^{2},\end{cases} \\
& \bar{v}(t)= \begin{cases}(-t+L \sqrt{-t}) e^{\lambda_{2}^{c} t}, & t<t_{2}, \\
L_{2}, & t \geq t_{2},\end{cases} \\
& \underline{v}(t)= \begin{cases}(-t-p \sqrt{-t}) e^{\lambda_{2}^{c} t}, & t<-p^{2}, \\
0, & t \geq-p^{2}\end{cases}
\end{aligned}
$$

by similar parameters in cases (1) and (2). By selecting large $L, p, q$, we may obtain the desirable inequalities. The proof is complete.

\section{Minimal Wave Speed}

In this part, we shall prove that $c^{*}$ is the minimal wave speed if

$$
a L_{2}<1 \text {, }
$$

which will be true throughout this section.

Theorem 3. If $c>(<) c^{*}$, then (21) and (22) have a strictly positive solution

$$
\begin{aligned}
& u \in C_{\left[0, r_{1}\right]}, \\
& v \in C_{\left[0, r_{2}\right]} .
\end{aligned}
$$

If $k_{1}, k_{2}$ admit the compact supports, then $c \geq c^{*}$ if and only if (21) and (22) have a strictly positive solution $u \in C_{\left[0, r_{1}\right]}, v \in C_{\left[0, r_{2}\right]}$.

In the following, we prove Theorem 3 by three lemmas.

Lemma 4. If (21) has a solution $u(t), v(t) \in C^{+}$such that

$$
\begin{aligned}
& u\left(t_{1}\right)>0, \\
& v\left(t_{2}\right)>0, \text { for some } t_{1}, t_{2} \in \mathbb{R},
\end{aligned}
$$

then

$$
\begin{array}{r}
L_{1} \geq u(t)>0, \\
L_{2} \geq v(t)>0, \\
t \in \mathbb{R} .
\end{array}
$$

The lemma is evident by (A) and the definition of traveling wave solutions, and we omit the proof. Clearly, this lemma implies a positive solution of (21) which is strictly positive. Furthermore, Lemma 4 implies the existence of nonconstant traveling wave solutions $u, v$ of (21) if $c \geq c^{*}$, which satisfy

$$
\begin{aligned}
\lim _{t \longrightarrow-\infty} u(t) & =\lim _{t \longrightarrow-\infty} v(t)=0, \\
0 & <u(t) \leq L_{1}, \\
0 & <v(t) \leq L_{2}, \\
t & \in \mathbb{R} .
\end{aligned}
$$

Therefore, we need to show the asymptotic behavior of traveling wave solutions when $t \longrightarrow \infty$ and the nonexistence of traveling wave solutions when $c<c^{*}$. On the limit behavior, we have the following conclusion.

Lemma 5. If $(u(t), v(t))$ is a positive solution of (21), then there exists $\delta>0$ such that

$$
\begin{aligned}
& \liminf _{t \longrightarrow-\infty} u(t)>\delta, \\
& \liminf _{t \longrightarrow-\infty} v(t)>\delta .
\end{aligned}
$$

Proof. By the definition, we see that $u(t)=u_{n}(x)$ satisfies

$$
\left\{\begin{array}{l}
u_{n+1}(x) \geq \int_{\mathbb{R}} u_{n}(y) e^{r_{1}\left(1-a L_{2}-u_{n}(y)\right)} k_{1}(x-y) \mathrm{d} y, \quad x \in \mathbb{R}, n=0,1,2, \ldots, \\
u_{0}(x)=u(x)>0, \quad x \in \mathbb{R} .
\end{array}\right.
$$


Let

$$
b(u)=\inf _{v \in\left[u, L_{1}\right]} v e^{r_{1}\left(1-a L_{2}-v\right)} .
$$

Then,

$\left\{\begin{array}{l}u_{n+1}(x) \geq \int_{\mathbb{R}} b\left(u_{n}(y)\right) k_{1}(x-y) d y, x \in \mathbb{R}, n=0,1,2, \ldots, \\ u_{0}(x)=u(x)>0, x \in \mathbb{R},\end{array}\right.$

and $b(u)$ is satisfied.

(C) $b:\left[0, L_{1}\right] \longrightarrow\left[0, L_{1}\right]$ is nondecreasing and continuous such that

$$
\lim _{u \longrightarrow 0^{+}} \frac{b(u)}{u}=e^{r_{1}\left(1-a L_{2}\right)}>1,
$$

and there exists $u_{*} \in\left(0, L_{1}\right]$ such that

$$
b(u)>u, u \in\left(0, u_{*}\right), b\left(u_{*}\right)=u_{*} .
$$

Due to Lemma 1, we have

$$
\liminf _{n \longrightarrow \infty,|x|<2 c} u_{n}(x) \geq u_{*}
$$

Since

$$
\bigcup_{n=0}^{\infty}(-2 c+c n, 2 c+c n)[0, \infty)
$$

we see that

$$
\liminf _{t \longrightarrow \infty} u(t) \geq u_{*}
$$

Similarly, we have

$$
\liminf _{t \rightarrow \infty} v(t)>\delta
$$

for some $\delta>0$. The proof is complete.

Lemma 6. If $c<c^{*}$, then (21) does not have a positive solution satisfying (22).

Proof. If the result is false for some $c<c_{2}^{*}$, then $v_{n}(x)=$ $v(x+c n)=v(t)$ satisfies

$$
\left\{\begin{array}{l}
v_{n+1}(x) \geq \int_{\mathbb{R}} v_{n}(y) e^{r_{2}\left(1-v_{n}(y)\right)} k_{2}(x-y) \mathrm{d} y, \quad x \in \mathbb{R}, n=0,1,2, \ldots, \\
v_{n}(x)=v(x)>0, \quad x \in \mathbb{R}, \\
\lim _{t \longrightarrow-\infty} v(t)=0 \\
\liminf _{t \longrightarrow \infty} v(t)>\delta
\end{array}\right.
$$

for some $\delta>0$. Let $-2 x(n)=\left(c+c_{2}^{*}\right) n$; then, $x(n)+c n \longrightarrow$ $-\infty$ if $n \longrightarrow \infty$.

Define

$$
b(u)=\inf _{v \in\left[u, L_{2}\right]} v e^{r_{2}(1-v)}, \quad u \in\left[0, L_{2}\right] .
$$

Then, $b(u)$ is satisfied.

(D) $b:\left[0, L_{2}\right] \longrightarrow\left[0, L_{2}\right]$ is nondecreasing and continuous such that

$$
\lim _{u \longrightarrow 0^{+}} \frac{b(u)}{u}=e^{r_{2}}>1,
$$

and there exists $v_{*} \in\left(0, L_{2}\right]$ such that

$$
\begin{aligned}
b(u) & >u, \\
u & \in\left(0, v_{*}\right), \\
b\left(v_{*}\right) & =v_{*} .
\end{aligned}
$$

From Lemma 1, we have

$$
\liminf _{n \rightarrow \infty} v_{n}\left(\frac{-\left(c+c_{2}^{*}\right) n}{2}\right) \geq v_{*} .
$$

Therefore, we obtain

$$
\liminf _{t \longrightarrow-\infty} v(t) \geq v_{*}>0,
$$

by $t=x+c n$. A contradiction occurs.

Similarly, we may prove that $c<c_{1}^{*}$ is impossible. The proof is complete.

Remark 3. If (8) and (59) hold, then we can obtain $\lim _{t \rightarrow \infty} u(t), \lim _{t \rightarrow \infty} v(t)$ by the simple fluctuation technique. In fact, let

$$
\begin{aligned}
& \liminf _{t \longrightarrow \infty} u(t)=\underline{u}, \\
& \liminf _{t \longrightarrow \infty} v(t)=\underline{v}, \\
& \limsup _{t \longrightarrow \infty} u(t)=\bar{u}, \\
& \limsup _{t \rightarrow \infty} v(t)=\bar{v} .
\end{aligned}
$$

Then, they are positive constants by Lemma 5, and the monotonicity and Fatou lemma further imply that

$$
\begin{aligned}
& 1-\bar{u}-a \underline{v} \geq 0, \\
& 1-\underline{u}-a \bar{v} \leq 0, \\
& 1-\bar{v}+b \bar{u} \geq 0, \\
& 1-\underline{v}+b \underline{u} \leq 0,
\end{aligned}
$$


and so $\underline{u}=\bar{u}, \underline{v}=\bar{v}$.

\section{Discussion}

Minimal wave speed of traveling wave solutions of evolutionary systems is very important in modeling the spatial expansion of individuals under consideration $[26,27]$. However, there are many open problems on the minimal wave speed of traveling wave solutions of different noncooperative systems. In this paper, we obtain the threshold for a predator-prey system without further monotone assumptions, which completes/improves the known results. We shall further utilize the techniques in this paper to more nonmonotone models including predator-prey systems and epidemic systems. For example, Lutscher ([15], Chapter 14) and Murray ([4], Sections 3.9 and 3.10) introduced some discrete time models; we will try to investigate the spatiotemporal modes of these models by developing the method in this paper.

In the literature, the minimal wave speed of traveling wave solutions may be the spreading speed of some systems $[14,16-21]$. Here, the spreading speed is an important threshold of the corresponding initial value problems with some special initial conditions [28]. For some monotone semiflows, it has been proved the equivalence of these thresholds, see Liang and Zhao [18]. However, for nonmonotone systems, very likely the above conclusion fails, and we may refer to two papers $[29,30]$ that studied a reaction-diffusion system of the predator-prey type. When the interspecific action between the predator and the prey is concerned, one typical problem is that the predator invades the habitat of the prey [31-34]. Due to the deficiency of the comparison principle, to further present the invasion threshold in such a nonmonotone system (9) is a challenging problem.

\section{Data Availability}

The data used to support the findings of this study are included within the article.

\section{Conflicts of Interest}

The authors declare that there are no conflicts of interest regarding the publication of this paper.

\section{Authors' Contributions}

All authors contributed equally to the writing of this paper. All authors read and approved the final manuscript.

\section{References}

[1] J. R. Beddington, C. A. Free, and J. H. Lawton, "Dynamic complexity in predator-prey models framed in difference equations," Nature, vol. 255, no. 5503, pp. 58-60, 1975.

[2] J. Hofbauer, V. Hutson, and W. Jansen, "Coexistence for systems governed by difference equations of Lotka-Volterra type," Journal of Mathematical Biology, vol. 25, no. 5, pp. 553-570, 1987.
[3] V. Weide, M. C. Varriale, F. M. Hilker, and F. M. Hilker, "Hydra effect and paradox of enrichment in discrete-time predator-prey models," Mathematical Biosciences, vol. 310, pp. 120-127, 2019.

[4] J. D. Murray, "Mathematical biology, I. An introduction," Interdisciplinary Applied Mathematics, Vol. 17, SpringerVerlag, New York, NY, USA, 3rd edition, 2002.

[5] W. E. Ricker, "Stock and recruitment," Journal of the Fisheries Research Board of Canada, vol. 11, no. 5, pp. 559-623, 1954.

[6] R. M. May, "Biological populations with nonoverlapping generations: stable points, stable cycles, and chaos," Science, vol. 186, no. 4164, pp. 645-647, 1974.

[7] R. M. May, "Simple mathematical models with very complicated dynamics," Nature, vol. 261, no. 5560, pp. 459-467, 1976.

[8] S. Ruan, "On nonlinear dynamics of predator-prey models with discrete delay," Mathematical Modelling of Natural Phenomena, vol. 4, no. 2, pp. 140-188, 2009.

[9] C. Carrillo and P. Fife, "Spatial effects in discrete generation population models," Journal of Mathematical Biology, vol. 50, no. 2, pp. 161-188, 2005.

[10] A. Bourgeois, V. LeBlanc, and F. Lutscher, "Spreading phenomena in integrodifference equations with nonmonotone growth functions," SIAM Journal on Applied Mathematics, vol. 78, no. 6, pp. 2950-2972, 2018.

[11] J. Jacobsen, Y. Jin, and M. A. Lewis, "Integrodifference models for persistence in temporally varying river environments," Journal of Mathematical Biology, vol. 70, no. 3, pp. 549-590, 2015.

[12] M. Kot and W. M. Schaffer, "Discrete-time growth-dispersal models," Mathematical Biosciences, vol. 80, no. 1, pp. 109-136, 1986.

[13] M. Kot, "Discrete-time travelling waves: ecological examples," J. Math. Biol.vol. 30, pp. 413-436, 1992.

[14] R. Lui, "Biological growth and spread modeled by systems of recursions. I. mathematical theory," Mathematical Biosciences, vol. 93, no. 2, pp. 269-295, 1989.

[15] F. Lutscher, Integrodifference Equations in Spatial Ecology, Springer, New York, NY, USA, 2019.

[16] H. F. Weinberger, "Long-time behavior of a class of biological models," SIAM Journal on Mathematical Analysis, vol. 13, no. 3, pp. 353-396, 1982.

[17] H. F. Weinberger, M. A. Lewis, and B. Li, "Analysis of linear determinacy for spread in cooperative models," Journal of Mathematical Biology, vol. 45, no. 3, pp. 183-218, 2002.

[18] X. Liang and X.-Q. Zhao, "Asymptotic speeds of spread and traveling waves for monotone semiflows with applications," Communications on Pure and Applied Mathematics, vol. 60, no. 1, pp. 1-40, 2007.

[19] S.-B. Hsu and X.-Q. Zhao, "Spreading speeds and traveling waves for nonmonotone integrodifference equations," SIAM Journal on Mathematical Analysis, vol. 40, no. 2, pp. 776-789, 2008.

[20] H. Wang and C. Castillo-Chavez, "Spreading speeds and traveling waves for non-cooperative integro-difference systems," Discrete and Continuous Dynamical Systems - Series B, vol. 17, no. 6, pp. 2243-2266, 2012.

[21] T. Yi, Y. Chen, and J. Wu, "Unimodal dynamical systems: comparison principles, spreading speeds and travelling waves," Journal of Differential Equations, vol. 254, no. 8, pp. 3538-3572, 2013.

[22] K. Li and X. Li, "The existence of traveling wave solutions in an integro-difference competition-cooperation system," 
International Journal of Biomathematics, vol. 11, no. 01, p. $1850009,2018$.

[23] G. Lin, "Traveling wave solutions for integro-difference systems," Journal of Differential Equations, vol. 258, no. 8, pp. 2908-2940, 2015.

[24] L. Li, S. Kang, L. Kong, and H. Chen, "Minimal wave speed of a competition integrodifference system," Journal of Difference Equations and Applications, vol. 24, no. 6, pp. 941-954, 2018.

[25] S. Pan, G. Lin, and J. Wang, "Propagation thresholds of competitive integrodifference systems," Journal of Difference Equations and Applications, vol. 25, no. 12, pp. 1680-1705, 2019.

[26] O. Diekmann, "Thresholds and travelling waves for the geographical spread of infection," Journal of Mathematical Biology, vol. 6, no. 2, pp. 109-130, 1978.

[27] J. D. Murray, "Mathematical biology, II. Spatial models and biomedical applications," Interdisciplinary Applied Mathematics, Vol. 18, Springer-Verlag, New York, NY, USA, 3rd edition, 2003.

[28] D. G. Aronson and H. F. Weinberger, "Nonlinear diffusion in population genetics, combustion, and nerve pulse propagation," in Partial Differential Equations and Related Topics, in: Lecture Notes in Math, J. A. Goldstein, Ed., vol. 446, pp. 5-49, Springer, Berlin, Germany, 1975.

[29] G. Lin, "Spreading speeds of a Lotka-Volterra predator-prey system: the role of the predator," Nonlinear Analysis: Theory, Methods \& Applications, vol. 74, no. 7, pp. 2448-2461, 2011.

[30] S. Pan, "Asymptotic spreading in a Lotka-Volterra predatorprey system," Journal of Mathematical Analysis and Applications, vol. 407, no. 2, pp. 230-236, 2013.

[31] A. Ducrot, "Convergence to generalized transition waves for some Holling-Tanner prey-predator reaction-diffusion system," Journal de Mathématiques Pures et Appliquées, vol. 100, no. 1, pp. 1-15, 2013.

[32] W. F. Fagan and J. G. Bishop, "Trophic interactions during primary succession: herbivores slow a plant reinvasion at Mount St. Helens," The American Naturalist, vol. 155, no. 2, pp. 238-251, 2000.

[33] M. Owen and M. A. Lewis, "How predation can slow, stop or reverse a prey invasion," Bulletin of Mathematical Biology, vol. 63, no. 4, pp. 655-684, 2001.

[34] S. Pan, "Invasion speed of a predator-prey system," Applied Mathematics Letters, vol. 74, pp. 46-51, 2017. 